\title{
Quantifying multipartite nonlocality via the size of the resource
}

\author{
Florian John Curchod, ${ }^{1, *}$ Nicolas Gisin, ${ }^{2}$ and Yeong-Cherng Liang ${ }^{3, \dagger}$ \\ ${ }^{1}$ ICFO-Institut de Ciències Fotòniques, 08860 Castelldefels (Barcelona), Spain \\ ${ }^{2}$ Group of Applied Physics, University of Geneva, CH-1211 Geneva 4, Switzerland \\ ${ }^{3}$ Institute for Theoretical Physics, ETH Zurich, 8093 Zurich, Switzerland
}

(Received 28 November 2014; published 30 January 2015)

\begin{abstract}
The generation of (Bell-)nonlocal correlations, i.e., correlations leading to the violation of a Bell-like inequality, requires the usage of a nonlocal resource, such as an entangled state. When given a correlation (a collection of conditional probability distributions) from an experiment or from a theory, it is desirable to determine the extent to which the participating parties would need to collaborate nonlocally for its (re)production. Here, we propose to achieve this via the minimal group size (MGS) of the resource, i.e., the smallest number of parties that need to share a given type of nonlocal resource for the above-mentioned purpose. In addition, we provide a general recipe-based on the lifting of Bell-like inequalities - to construct MGS witnesses for nonsignaling resources starting from any given ones. En route to illustrating the applicability of this recipe, we also show that when restricted to the space of full-correlation functions, nonsignaling resources are as powerful as unconstrained signaling resources. Explicit examples of correlations where their MGS can be determined using this recipe and other numerical techniques are provided.
\end{abstract}

DOI: 10.1103/PhysRevA.91.012121

PACS number(s): 03.65.Ud, 03.67.Mn, 03.67.Hk

\section{INTRODUCTION}

Quantum correlations that violate a Bell-type inequality [1], a constraint that was first derived in the studies of local-hidden-variable theories, were initially perceived only as a counterintuitive feature that has no classical analog. Following the discovery of quantum information science, these bizarre correlations have taken the new role as a resource. For instance, in the context of nonlocal games [2] (which are closely related to the studies of interactive proof systems in complexity theory; see, e.g., Ref. [3]), nonlocal, i.e., Bell-inequality-violating correlations are those that cannot be simulated by shared randomness (SR). They are also well known as an indispensable resource in quantum information processing and communication tasks such as the reduction of communication complexity [4], the distribution of secret keys in a device-independent setting [5], as well as the certification and expansion of randomness [6], etc. For a comprehensive review on these and other applications, see Ref. [7].

As in any other resource theory [8], the interconvertibility of resources, and the possibility to substitute one by another in a certain task are important ingredients that put our understanding of these resources on a firm ground. Considerable effort has been devoted to these questions in the bipartite setting -in the cost of simulating quantum correlations using classical communication [9] or certain "nonlocal boxes" [10], as well as the interconvertibility between these different resources [11,12] (see Ref. [7] for a review). However, relatively little is known $[13,14]$ in the multipartite scenarios, where other interesting features are also present, such as the monogamy of nonlocal correlations (see, e.g., Refs. [7,15] and references therein) and the possibility of them being anonymous [16].

\footnotetext{
*florian.curchod@icfo.es

†yliang@phys.ethz.ch
}

Thus far, prior investigations on multipartite nonlocal correlations have focused predominantly on their $m$ separability, namely, the possibility to reproduce them when the parties are separated into $m$ groups [17]—-specifically two groups [18-23] — and where the usage of some nonlocal resource $\mathcal{R}$ is only allowed within each group. ${ }^{1}$ While this has been a fruitful approach for the detection of genuine multipartite nonlocality, and hence genuine multipartite entanglement in a deviceindependent setting [20,24-26], it is, however, not always applicable to the detection of genuine multipartite nonlocality among a subset of participating parties. To manifest this shortcoming, let us consider a 4-partite correlation $\vec{P}=$ $\{P(\vec{a} \mid \vec{x})\}=\left\{P\left(a_{1} a_{2} a_{3} a_{4} \mid x_{1} x_{2} x_{3} x_{4}\right)\right\}$ of getting measurement outcome (output) $a_{i}$ for the $i$ th party given the measurement setting (input) $x_{i}$. A specific kind of biseparable correlation in this scenario takes the form of

$$
\begin{aligned}
P(\vec{a} \mid \vec{x})= & \sum_{\lambda} q_{\lambda} P_{\lambda}^{\mathcal{R}}\left(a_{1} a_{2} \mid x_{1} x_{2}\right) P_{\lambda}^{\mathcal{R}}\left(a_{3} a_{4} \mid x_{3} x_{4}\right) \\
& +\sum_{\mu} q_{\mu} P_{\mu}^{\mathcal{R}}\left(a_{1} a_{3} \mid x_{1} x_{3}\right) P_{\mu}^{\mathcal{R}}\left(a_{2} a_{4} \mid x_{2} x_{4}\right) \\
& +\sum_{\nu} q_{\nu} P_{\nu}^{\mathcal{R}}\left(a_{1} a_{4} \mid x_{2} x_{3}\right) P_{\nu}^{\mathcal{R}}\left(a_{2} a_{3} \mid x_{2} x_{3}\right)
\end{aligned}
$$

where $P_{i}^{\mathcal{R}}\left(a_{j} a_{k} \mid x_{j} x_{k}\right)$ is some 2-partite distribution allowed by the resource $\mathcal{R}$, while $q_{\lambda}, q_{\mu}$, and $q_{\nu}$ are non-negative, normalized weights. If $\vec{P}$ cannot be written in the form of Eq. (1), the production of this correlation clearly requires at least three out of the four participating parties to collaborate nonlocally via $\mathcal{R}$. If, moreover, nonlocal collaboration between three parties is sufficient, we see that $\vec{P}$ is thus biseparable, i.e., producible by parties separated into (convex mixtures of) two groups; see Fig. 1. In other words, the

\footnotetext{
${ }^{1}$ However, we do assume that global shared randomness is available for free in the resource theory of correlations.
} 


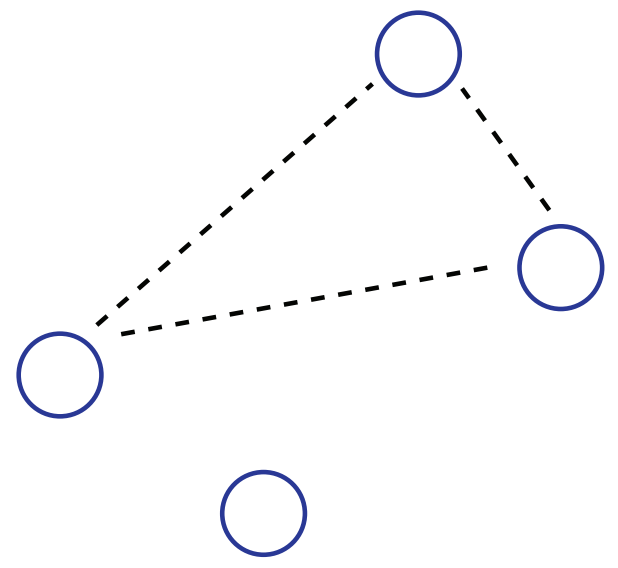

FIG. 1. (Color online) Schematic diagram showing a situation where the conventional approach of nonbiseparability fails to detect the multipartite nonlocality present in the correlation. Here, the dashed lines joining the three circles symbolically represent the nonlocal collaboration between the three parties. Since the fourth party is only correlated with the rest through shared randomness, the overall correlation is biseparable.

multipartite nonlocality contained in $\vec{P}$ cannot be detected by the conventional approach of detecting nonbiseparability. Indeed, with the conventional ( $m$-separability) approach, one only makes a distinction between the number of groups, but not the size, i.e., the number of parties involved in each group.

To determine the extent to which participating parties would need to collaborate nonlocally in a general scenario, it thus seems more natural to quantify multipartite nonlocality in terms of the minimal group size (MGS), i.e., the smallest number of parties required to collaborate nonlocally in reproducing some nonlocal correlation. Clearly, this approach provides information complementary to the one of $m$ separability on how $\mathcal{R}$ has to be distributed and/or shared among the participating parties in order to reproduce some given correlation. The aim of this paper is to give a state-of-the-art exposition of this approach and to provide a general technique for the construction of MGS witnesses.

The rest of this paper is structured as follows. In Sec. II, we give a more formal introduction to the notion of MGS and the closely related concept of $k$ producibility; their connection to the conventional notion of genuine multipartite nonlocality is also discussed therein. Then in Sec. II A, we give an exposition of some basic facts about the sets of correlations that are $k$ producible. After that, in Secs II B and IIC, we give examples of quantum-realizable correlations where their characterization via the MGS approach is both natural and explicit. In Sec. III, we provide a general recipe to construct $n$-partite witness of non- $k$-producibility-i.e., witnesses certifying MGS $>k$-starting from any given witness involving a smaller number of parties. There, we also make a digression to point out the universality of nonsignaling resource when one is only concerned with the so-called full-correlation functions [20]. Finally, we conclude with some possible future research in Sec. IV. Proofs of the two theorems and one corollary given in Sec. III are relegated to the Appendixes.

\section{MINIMAL GROUP SIZE, $k$ PRODUCIBILITY, AND MULTIPARTITE NONLOCALITY}

Formally, let us remind that an $n$-partite correlation $\vec{P}=\{P(\vec{a} \mid \vec{x})\}$ is a collection of the conditional probability distributions of getting outputs $\vec{a}=\left(a_{1}, a_{2}, \ldots, a_{n}\right)$, given the inputs $\vec{x}=\left(x_{1}, x_{1}, \ldots, x_{n}\right)$. In analogy with the studies of multipartite entanglement [27], we say that $\vec{P}$ is $k$ producible (or more precisely $k$-partite $\mathcal{R}$ producible) if

(1) $\vec{P}$ can be decomposed into a convex mixture of products of at most $k$-partite correlations, and

(2) each constituent correlation satisfies the constraints defined by the resource $\mathcal{R} .^{2}$

As a basic example, we note that by definition, a correlation $\vec{P}$ is Bell-local (henceforth local) if

$$
P(\vec{a} \mid \vec{x})=\sum_{\lambda} q_{\lambda} \prod_{i=1}^{n} P_{\lambda}\left(a_{i} \mid x_{i}\right),
$$

for some choice of normalized weights $q_{\lambda} \geqslant 0$ and some constituent correlations $P_{\lambda}\left(a_{i} \mid x_{i}\right)$. A local correlation is thus 1-producible, and hence producible by each party being alone and sharing no nonlocal resource with the others. On the other hand, correlation satisfying Eq. (1) is 2-producible whereas a correlation $\vec{P}$ satisfying

$$
\begin{aligned}
P(\vec{a} \mid \vec{x})= & \sum_{\lambda} q_{\lambda} P_{\lambda}^{\mathcal{R}}\left(a_{1} a_{2} a_{3} \mid x_{1} x_{2} x_{3}\right) P_{\lambda}^{\mathcal{R}}\left(a_{4} \mid x_{4}\right) \\
& +\sum_{\mu} q_{\mu} P_{\mu}^{\mathcal{R}}\left(a_{1} a_{2} a_{4} \mid x_{1} x_{2} x_{4}\right) P_{\mu}^{\mathcal{R}}\left(a_{3} \mid x_{3}\right) \\
& +\sum_{\nu} q_{\nu} P_{\nu}^{\mathcal{R}}\left(a_{1} a_{3} a_{4} \mid x_{1} x_{3} x_{4}\right) P_{\nu}^{\mathcal{R}}\left(a_{2} \mid x_{2}\right) \\
& +\sum_{\theta} q_{\theta} P_{\theta}^{\mathcal{R}}\left(a_{2} a_{3} a_{4} \mid x_{2} x_{3} x_{4}\right) P_{\theta}^{\mathcal{R}}\left(a_{1} \mid x_{1}\right)
\end{aligned}
$$

is 3-producible. A general 3-producible correlation, however, may involve a convex combination of correlation of the form of Eq. (1) and of Eq. (3). Obviously, $k$ producibility implies $k^{\prime}$ producibility for all $k^{\prime}>k$. Using the above terminologies, we thus say that $\vec{P}$ is genuinely $k$-partite nonlocal ${ }^{3}$ or having a MGS of $k$ if $\vec{P}$ is $k$ producible but not $(k-1)$ producible. For example, a 4-partite correlation that satisfies Eq. (1) but not Eq. (2) is 2-producible but not 1-producible, and hence genuinely 2-partite nonlocal. Similarly, a 4-partite correlation that is 3-producible but not decomposable in the form of Eq. (1) is genuinely 3 -partite nonlocal.

A few other remarks are now in order. First, the above definition can be seen as a generalization of existing notions of genuine $k$-partite nonlocality for an $n=k$-partite scenario

\footnotetext{
${ }^{2}$ For example, if $\mathcal{R}$ refers to a quantum resource, then the constituent correlation must be producible by performing local measurements on some quantum state.

${ }^{3}$ To conform with existing terminologies in the literature, when $\mathcal{R}$ refers to a quantum resource, we say that $\vec{P}$ must have arisen from a genuinely $k$-partite entangled state instead of $\vec{P}$ exhibits genuine $k$-partite nonlocality.
} 
[23] to an $n$-partite scenario where $n \geqslant k$. It is worth noting that the question of whether a given correlation $\vec{P}$ can be produced by having at most $k$ parties in one group ( $k$ producibility) is not completely independent from the question of whether $\vec{P}$ can be produced by separating the $n$ parties into at least $m$ groups ( $m$ separability). For instance, a $k$-producible correlation $\vec{P}$ is $m$ separable for some $m \geqslant\left\lceil\frac{n}{k}\right\rceil$; likewise, if $\vec{P}$ is $m$ separable, it is also $k$ producible for some $k \geqslant\left\lceil\frac{n}{m}\right\rceil$. Thus, the smallest $n$ for which these descriptions become inequivalent is $n=4$. Finally, any multipartite correlation that cannot be produced by SR, or equivalently that is nonlocal [cf. Eq. (2)], or not 1 -producible, is genuinely $k$-partite nonlocal for some $k \geqslant 2$.

\section{A. Characterization of the sets of $\boldsymbol{k}$-partite $\mathcal{R}$-producible correlations}

While the bulk of the above discussion is independent of the choice of the nonlocal resource $\mathcal{R}$, it is worth reminding some features that are pertinent to specific resources. In this context, four commonly discussed nonlocal resources $\mathcal{R}$ are (1) $\mathcal{Q}$ : (local measurements on) an entangled quantum state of unrestricted Hilbert space dimension; (2) $\mathcal{N S}$ : a post-quantum, but nonsignaling $[11,28]$ resource $^{4}(3) \mathcal{T}$ [22,23]: a timeordered, one-way classical signaling resource, ${ }^{5}$ and (4) $\mathcal{S}$ [18]: a Svetlichny resource. ${ }^{6}$ Note that each resource $\mathcal{R}$ above is strictly stronger than the preceding one(s), in the sense that $\mathcal{R}$ can be used to produce all correlations arising from the preceding resource(s) $[22,23]$. As a result, we have the strict inclusion relations,

$$
\mathcal{L} \subset \mathcal{Q} \subset \mathcal{N S} \subset \mathcal{T} \subset \mathcal{S},
$$

with $\mathcal{L}$ being a local resource, provided by SR alone. Hence, a correlation $\vec{P}$ that is $k$-partite $\mathcal{Q}$ producible is also a member of the set of $k$-partite $\mathcal{R}$-producible correlations (henceforth denoted by $\mathcal{R}_{n, k}$ ) for $\mathcal{R} \in\{\mathcal{N} \mathcal{S}, \mathcal{T}, \mathcal{S}\}$. Conversely, a correlation that is not in $\mathcal{S}_{n, k}$ is also not in $\mathcal{R}_{n, k}$ for $\mathcal{R} \in\{\mathcal{Q}, \mathcal{N} \mathcal{S}, \mathcal{T}\}$; see Fig. 2. Formally, these implications are summarized as follows:

$$
\begin{gathered}
\vec{P} \in \mathcal{Q}_{n, k} \Rightarrow \vec{P} \in \mathcal{R}_{n, k} \quad \text { for all } \quad \mathcal{R} \in\{\mathcal{N} \mathcal{S}, \mathcal{T}, \mathcal{S}\}, \\
\vec{P} \notin \mathcal{S}_{n, k} \Rightarrow \vec{P} \notin \mathcal{R}_{n, k} \quad \text { for all } \quad \mathcal{R} \in\{\mathcal{Q}, \mathcal{N} \mathcal{S}, \mathcal{T}\} .
\end{gathered}
$$

More generally, we note that independent of the nonlocal resource $\mathcal{R} \in\{\mathcal{Q}, \mathcal{N} \mathcal{S}, \mathcal{T}, \mathcal{S}\}$, the set $\mathcal{R}_{n, k}$ is convex. Moreover, for the case when $\mathcal{R} \in\{\mathcal{N} \mathcal{S}, \mathcal{T}, \mathcal{S}\}, \mathcal{R}_{n, k}$ is even a convex

\footnotetext{
${ }^{4}$ Such a resource only allows correlations where their marginal distributions for any subset of parties are independent of the input of the complementary subset of parties.

${ }^{5}$ The correlations allowed by such a resource is referred to as timeordered bilocal in Ref. [22].

${ }^{6}$ The Svetlichny resource allows the parties in a group to use any joint strategy and hence to produce any correlation that is only constrained by the normalization of probabilities. In some cases, such a resource can be realized by allowing multiple rounds of classical communications among the parties but in others, such a resource may not have a well-defined physical meaning; see Refs. [22,23] for a discussion.
}

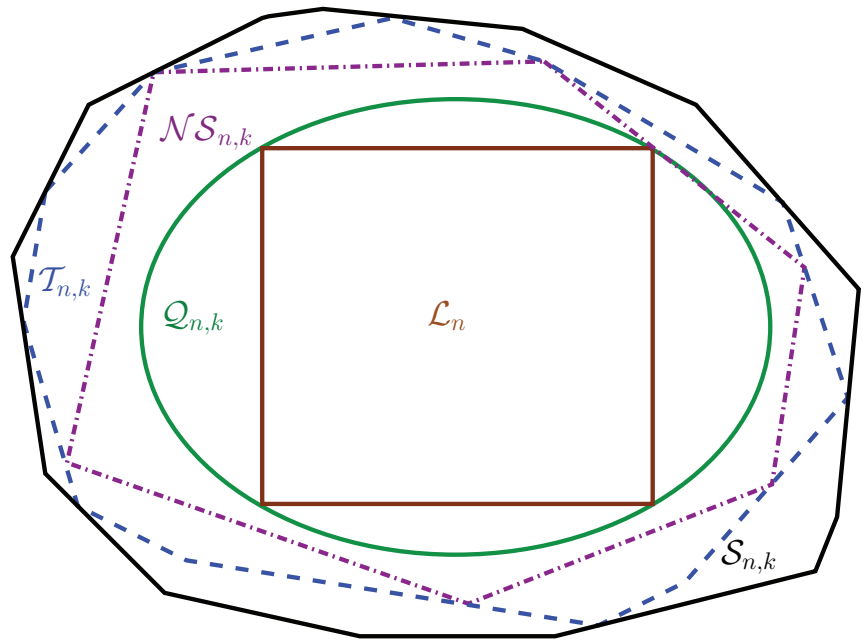

FIG. 2. (Color online) Schematic diagram showing the inclusion relations of the various sets of $\mathcal{R}_{n, k}$; cf. Eqs. (4) and (5). The smallest of these sets is $\mathcal{L}_{n}$ [depicted as the (brown) rectangle], followed by $\mathcal{Q}_{n, k}$ [depicted as the (green) oval], followed by $\mathcal{N S}_{n, k}$ [with boundary marked by the (magenta) dashed-dotted line], followed by $\mathcal{T}_{n, k}$ [with boundary marked by the (blue) dashed line]. Finally, the $k$-producible Svetlichny set $\mathcal{S}_{n, k}$ is represented by the outermost (black) solid polygon.

polytope [23], i.e., a convex set having only a finite number of extreme points [29] and thus can be equivalently specified through a finite number of Bell-like inequalities (corresponding to the facets of the respective polytope). Determining if a given correlation $\vec{P}$ is inside $\mathcal{R}_{n, k}$, and hence producible by the respective resource can thus be decided via a linear program [30], or through the violation of one of those Bell-like inequalities defining the polytope. In the simplest 2-input, 2-output scenario where $\mathcal{R}=\mathcal{N} \mathcal{S}$, the set $\mathcal{N S}_{3,2}$ has been completely characterized in Ref. [23] whereas a superset of $\mathcal{N S}_{4,2}$ has also been characterized in Ref. [31] (see also Ref. [32]). If $\mathcal{R}=\mathcal{Q}$, i.e., a quantum resource, then the set $\mathcal{R}_{n, k}$ is no longer a convex polytope. Determining if a given $\vec{P}$ is in $\mathcal{Q}_{n, n-1}$ can nonetheless be achieved by solving a hierarchy of semidefinite programs [30] described in Ref. [24]. More generally, determining if any given $\vec{P}$ is in $\mathcal{Q}_{n, k}$ can be achieved - to some extent - by solving a variant of the hierarchy of semidefinite programs described in Ref. [26] (see Ref. [33] for details).

However, regardless of $\mathcal{R}$, it is generally formidable to solve the aforementioned linear or semidefinite programs by brute force even on a computer for relatively simple scenarios. Implications such as those summarized in Eq. (5) are thus useful to bear in mind for subsequent discussions. For example, if $\vec{P}$ violates an $n$-partite Svetlichny inequality-a Bell-like inequality that holds for a general Svetlichny resource-then it is not $(n-1)$ producible for all $\mathcal{R}$. In other words, the correlation $\vec{P}$ exhibits genuine $n$-partite nonlocality (and hence can only be produced, if at all, by a genuinely $n$-partite entangled state) and has an MGS of $n$. A generic correlation $\vec{P}$, evidently, will have an MGS that depends on the resource under consideration, as we now illustrate by explicit examples in the following subsections. 


\section{B. An example of a genuinely 3-partite $\mathcal{N} \mathcal{S}$ nonlocal correlation in a 4-partite scenario}

The Greenberger-Horne-Zeilinger (GHZ) state [34] between $n$ parties is defined as follows:

$$
\left|\mathrm{GHZ}_{\mathrm{n}}\right\rangle=\frac{1}{\sqrt{2}}\left(|0\rangle^{\otimes \mathrm{n}}+|1\rangle^{\otimes \mathrm{n}}\right),
$$

where $|0\rangle$ and $|1\rangle$ are, respectively, the eigenstate of the Pauli matrix $\sigma_{z}$ with eigenvalue +1 and -1 . Consider the following equal-weight mixture of three parties sharing $\left|\mathrm{GHZ}_{3}\right\rangle$ and one party holding $|-\rangle$ :

$$
\rho=\frac{1}{4}\left(\left|\mathrm{GHZ}_{3}\right\rangle\left\langle\mathrm{GHZ}_{3}|\otimes|-\right\rangle-\mid+\circlearrowright\right),
$$

where $|-\rangle$ is the eigenstate of the Pauli matrix $\sigma_{x}$ with eigenvalue -1 , and we have used $\circlearrowright$ to denote similar terms which must be included to ensure that the expression involved is invariant under arbitrary permutation of parties. This quantum state could be prepared, for instance, by distributing uniformly randomly $\left|\mathrm{GHZ}_{3}\right\rangle$ to any of the three parties and $|-\rangle$ to the remaining one. By construction, $\rho$ does not have genuine 4-partite entanglement. Hence, any correlation $\vec{P}$ derived by performing local measurement on $\rho$ must be a member of $\mathcal{Q}_{4,3}$ and by Eq. (5a), also $\mathcal{R}_{4,3}$.

Now, consider the case where all parties measure the following dichotomic observables,

$$
\begin{aligned}
& A_{0}=B_{0}=C_{0}=D_{0}=-\frac{\sqrt{3}}{2} \sigma_{x}+\frac{1}{2} \sigma_{y}, \\
& A_{1}=B_{1}=C_{1}=D_{1}=-\frac{\sqrt{3}}{2} \sigma_{x}-\frac{1}{2} \sigma_{y} .
\end{aligned}
$$

It can be shown that that the resulting correlation $\vec{P}$ violates the following Bell inequality which must be satisfied by all correlations from $\mathcal{N S}_{4,2}$ [31]:

$$
\begin{aligned}
\mathcal{I}= & -12\left\langle A_{0}\right\rangle-3\left\langle A_{1}\right\rangle-2\left\langle A_{0} B_{0}\right\rangle+6\left\langle A_{0} B_{1}\right\rangle \\
& -3\left\langle A_{1} B_{1}\right\rangle+13\left\langle A_{0} B_{0} C_{0}\right\rangle-3\left\langle A_{1} B_{0} C_{0}\right\rangle \\
& -11\left\langle A_{1} B_{1} C_{0}\right\rangle+14\left\langle A_{1} B_{1} C_{1}\right\rangle+22\left\langle A_{0} B_{0} C_{0} D_{0}\right\rangle \\
& -15\left\langle A_{0} B_{0} C_{0} D_{1}\right\rangle-10\left\langle A_{1} B_{1} C_{0} D_{0}\right\rangle \\
& -7\left\langle A_{1} B_{1} C_{1} D_{0}\right\rangle+21\left\langle A_{1} B_{1} C_{1} D_{1}\right\rangle+\circlearrowright \\
\mathcal{N} \mathcal{S}_{4,2} &
\end{aligned}
$$

giving a quantum value of 117.8827 . This implies that the correlation $\vec{P}$ is also genuinely 3 -partite nonlocal, or having an MGS of 3 for $\mathcal{R} \in\{\mathcal{Q}, \mathcal{N S}\}$.

Interestingly, it can be shown that $\vec{P}$ does not lie in any of the 3 -partite $\mathcal{N S}$-producible sets corresponding to a fixed partition. This, together with the fact that $\stackrel{\vec{P}}{\text { is }}$ 3-partite $\mathcal{N S}$ producible means that the generation of $\vec{P}$ requires classical mixtures of different partitions of the four participating parties into two groups, one of them containing three parties and sharing an $\mathcal{N S}$ resource. It is also worth noting that all tripartite marginal correlations of $\vec{P}$ are verifiably 1-producible (hence satisfying the complete set of Bell inequalities for this scenario given in Ref. [35]). In other words, although $\vec{P}$ is genuinely 3-partite $\mathcal{N S}$ nonlocal, this 3 -partite nonlocality cannot be revealed by studying each of the four tripartite marginal correlations individually. Neither can this multipartite nonlocality be manifested by analyzing the biseparability of the 4-partite correlation since this more conventional approach cannot distinguish correlation of the form of Eq. (1) and those of the form of Eq. (3).

In the above example, we were able to determine the MGS of the correlation for the quantum, and a general nonsignaling resource. For the Svetlichny resource, we could also show-by solving some linear program - that the very same correlations is inside the set $\mathcal{S}_{4,2}$, and thus only exhibits an MGS of 2 . However, due to the computational complexity involved in solving the corresponding linear program for the one-way signaling resource $\mathcal{T}$, we were not able to determine precisely its MGS. Apart from correlations that violate an $n$-partite Svetlichny inequality (in which case MGS $=n$ for all resources) or correlations that are local (in which case MGS $=1$ ), one may thus wonder if there exist other $n$-partite correlations $\vec{P}$ which have an MGS that can be fully characterized for all the different resources. We now provide examples of this kind in the next section.

\section{A family of $\boldsymbol{n}$-partite examples with fully characterized MGS}

In Ref. [16], it has been shown that if all $n$ parties either measure the $\sigma_{x}$ or the $\sigma_{y}$ observable on the $n$-partite state $\left|\mathrm{GHZ}_{\mathrm{n}}\right\rangle$, Eq. (6), the resulting correlation has an MGS of $\left\lceil\frac{n}{2}\right\rceil$ for $\mathcal{R} \in\{\mathcal{N S}, \mathcal{T}, \mathcal{S}\}$ whenever $n$ is odd or $\frac{n}{2}$ is even. On the other hand, if we restrict ourselves to a quantum resource, then for all odd $n \geqslant 3$, it follows from the result of Ref. [16] that the corresponding MGS is $n$, demonstrating a large gap between the size of the resource required to reproduce these correlations when using a quantum and a post-quantum nonsignaling (or a classical but signaling) resource. To prove these results, a general $\mathcal{N S}$ biseparable decomposition of the aforementioned correlation was provided [16] for arbitrary partitioning of the $n$ parties into two groups, thus establishing that these correlations are $\left\lceil\frac{n}{2}\right\rceil$ producible for $\mathcal{R} \in\{\mathcal{N} \mathcal{S}, \mathcal{T}, \mathcal{S}\}$. Then to prove that these correlations are not $\left(\left\lceil\frac{n}{2}\right\rceil-1\right)$ producible for the same set of resources, it was shown in Ref. [16] that except for even $n$ with odd $\frac{n}{2}$, these correlations are not 3 -separable, i.e., cannot be reproduced by a separation of the $n$ parties into three groups. As for $\mathcal{R}=\mathcal{Q}$, an $\mathrm{MGS}=n$ for odd $n$ [16] follows from the fact that the corresponding correlation violates a device-independent witness for genuine $n$-partite entanglement [20,24] constructed from the Mermin-ArdehaliBelinskii-Klyshko (MABK) Bell expression [36,37]. In the case of even $n$, the result recently established in Ref. [33] (based on earlier work of Ref. [38]) allows one to conclude that the above-mentioned GHZ correlations have an MGS of at least $n-1$ (for $\mathcal{R}=\mathcal{Q}$ ).

\section{WITNESSING NON- $k$-PRODUCIBILITY USING BELL-LIKE INEQUALITIES}

Evidently, as discussed in Sec. II, Bell-like inequalities are very useful tools for determining (or at least lower bounding) the MGS of a given correlation by certifying its non- $k$-producibility. For example, all Bell-like inequalities that have been derived-based on the nonbiseparability approach [18-23] - to detect genuine $n$-partite nonlocality can be used 
as witnesses for non- $(n-1)$-producibility for the respective resources. It is, however, unrealistic to hope to find all such Bell-like inequalities by solving the polytope describing the convex set $\mathcal{R}_{n, k}$ even for relatively small $n$ and $k$. But all is not lost and in this section, we recall from Ref. [39] the technique of lifting — originally developed for Bell inequalities that witness Bell-nonlocality-and show that it can also be used to construct Bell-like inequality for arbitrary $\mathcal{R}_{n^{\prime}, k}$ (where $n^{\prime}>n$ and $\mathcal{R} \in\{\mathcal{Q}, \mathcal{N} \mathcal{S}\}$ ) starting from any given Bell-like inequality for $\mathcal{R}_{n, k}$. Before that, let us first make a digression and point out the usefulness of a nonsignaling resource in simulating a general correlation.

\section{A. All extremal full-correlation functions can be simulated with nonsignaling strategies}

In an $n$-partite, $m$-input, $\ell$-output Bell scenario, the set of full-correlation functions defined in Ref. [20] consists of the following $\ell \mathrm{m}^{n}$ joint conditional probability distributions:

$$
\left\{P\left(\left[\mathbf{a}_{\vec{x}}\right]_{\ell}=r\right)\right\}_{r=0}^{\ell-1},
$$

where $[X]_{\ell}:=X \bmod \ell$,

$$
P\left(\left[\mathbf{a}_{\vec{x}}\right]_{\ell}=r\right)=\sum_{\vec{a}} P(\vec{a} \mid \vec{x}) \delta_{\sum_{i} a_{i} \bmod \ell, r},
$$

and $\delta_{a, b}$ is the Kronecker delta of $a$ and $b$. Note that due to the normalization conditions, only $(\ell-1) m^{n}$ of these joint conditional probability distributions are independent. Moreover, in the case where there are only two possible outcomes, i.e., $\ell=2$, it is easy to see that the above definition of full-correlation functions is equivalent to the conventional one defined by the expectation value of the product of \pm 1 outcomes.

We now present a mathematical fact about the space of correlations spanned by the set of full-correlation functions defined in Eq. (10).

Theorem 1. When restricted to the set of full-correlation functions given in Eq. (10), all extremal strategies achievable by an $n$-partite Svetlichny resource $\mathcal{S}_{n}$ are also achievable using an $n$-partite nonsignaling resource $\mathcal{N} \mathcal{S}_{n}$. Thus, in the subspace spanned by full-correlation functions, the three sets of correlations $\mathcal{S}_{n}, \mathcal{I}_{n}$, and $\mathcal{N S}$ become identical.

One can find the proof of this theorem in Appendix A. Let us remember that the Svetlichny resource is the most powerful nonlocal resource, and is only constrained by the normalization of probability distributions. In other words, $\mathcal{S}_{n}$ is basically the set of normalized $n$-partite correlations. The importance of Theorem 1 is that when restricted to the set of coarse-grained measurement statistics represented by the set of full-correlation functions, cf. Eq. (10a), one also cannot make a distinction between $\mathcal{N S}_{n}$ and the set of normalized conditional probability distributions. Note that for binary-outcome full-correlation functions arising from the Bell singlet state, the coincidence between $\mathcal{S}_{2}$ and $\mathcal{N} \mathcal{S}_{2}$ was already anticipated from the results of Ref. [10]. In fact, an alternative proof of Theorem 1 for the special case of binary-outcome full-correlation functions can be found, e.g., in Theorem 12 of Ref. [40].
It is also worth noting that the definition of full-correlation functions is not unique, nevertheless, numerous Bell-like inequalities can be written in terms of the correlation functions defined in Eq. (10); see e.g., Ref. [20]. In this regard, note also the following corollary of Theorem 1 , which allows us to relate Bell-like inequalities for $\mathcal{N} \mathcal{S}_{n, k}$ with those of $\mathcal{R}_{n, k}$ for $\mathcal{R} \in\{\mathcal{T}, \mathcal{S}\}$.

Corollary 1. Let $\mathcal{I}_{n, k}^{\mathcal{R}}$ be a tight, full-correlation Bell-like inequality that holds for $\mathcal{R} \in\{\mathcal{T}, \mathcal{S}\}$, i.e.,

$$
\mathcal{I}_{n, k}^{\mathcal{R}}: \sum_{\vec{x}} \sum_{r=0}^{\ell-1} \beta_{\vec{x}}^{r} P\left(\left[\mathbf{a}_{\vec{x}}\right]_{\ell}=r\right) \stackrel{\mathcal{R}_{n, k}}{\leqslant} B_{n, k}^{\mathcal{R}},
$$

and there exists $P(\vec{a} \mid \vec{x}) \in \mathcal{R}_{n, k}$ such that inequality (11) becomes an equality, then there also exists $P(\vec{a} \mid \vec{x}) \in \mathcal{N} \mathcal{S}_{n, k}$ such that inequality (11) becomes an equality. In other words,

$$
\sum_{\vec{x}} \sum_{r=0}^{\ell-1} \beta_{\vec{x}}^{r} P\left(\left[\mathbf{a}_{\vec{x}}\right]_{\ell}=r\right) \stackrel{\mathcal{N} \mathcal{S}_{n, k}}{\leqslant} B_{n, k}^{\mathcal{R}},
$$

is also a tight, full-correlation Bell-like inequality that holds for $\mathcal{R}=\mathcal{N S}$.

The proof of the above corollary can be found in Appendix B. The corollary tells us that if we restrict ourselves to Belllike inequalities that only involve linear combination of fullcorrelation functions, Eq. (10), then we cannot distinguish between correlations that are $k$ producible with respect to any of the resource $\mathcal{R} \in\{\mathcal{N S}, \mathcal{T}, \mathcal{S}\}$. In other words, for any given $n$ and $k$ and in the subspace of measurement statistics spanned by the set of full-correlation functions, cf. Eq. (10), the three sets of correlations $\mathcal{N} \mathcal{S}_{n, k}, \mathcal{T}_{n, k}$, and $\mathcal{S}_{n, k}$ become identical. It is worth bearing this fact in mind in order to appreciate the generality of the upcoming theorem.

\section{B. Lifting of Bell-like inequalities}

The lifting of Bell inequalities was first discussed by Pironio in Ref. [39]. Essentially, it is a technique that allows one to extend any (facet-defining) Bell inequality of a given scenario to a more complex scenario (involving more parties and/or inputs and/or outputs). In this work, we are only interested in the lifting of Bell-like inequalities to a scenario involving more parties. In this case, a lifted Bell inequality corresponds to a witness of nonlocality where the nonlocal behavior of a subset of, say $n$, of the parties becomes apparent after conditioning on a specific combination of measurement settings and outcomes from the complementary subset of $h$ parties. $^{7}$

More concretely, let us denote a specific combination of the measurement settings and measurement outcomes of the $h$ parties, respectively, by $\vec{s}$ and $\vec{o}$. It can then be shown that if the $(n+h)$-partite correlation $P(\vec{a}, \vec{o} \mid \vec{x}, \vec{s})$ is 1-producible (and

\footnotetext{
${ }^{7}$ This particular kind of lifting has been applied to show, for instance, a stronger version of Bell's theorem; see, e.g., Ref. [41].
} 
nonvanishing ${ }^{8}$ ), so is the conditional distribution given by

$$
\tilde{P}^{\mid \vec{o}, \vec{s}}(\vec{a} \mid \vec{x})=\frac{P(\vec{a}, \vec{o} \mid \vec{x}, \vec{s})}{\sum_{\vec{a}} P(\vec{a}, \vec{o} \mid \vec{x}, \vec{s})}
$$

An immediate implication of this is that a Bell inequality that is defined for an $n$-partite scenario can be trivially extended to any $(n+h)$-partite scenarios by considering specific measurement settings $\vec{s}$ and outcomes $\vec{o}$ for the $h$ parties.

As an example consider the well-known Clauser-HorneShimony-Holt [42] Bell inequality applicable to a scenario involving two parties, each performing two binary-outcome measurements:

$$
\sum_{x_{1}, x_{2}, a_{1}, a_{2}=0}^{1}(-1)^{a_{1}+a_{2}+x_{1} x_{2}} P\left(a_{1} a_{2} \mid x_{1} x_{2}\right) \stackrel{\mathcal{L}}{\leqslant} 2 .
$$

Lifting this inequality to the scenario of three parties and with the third party getting a specific measurement outcome $o_{3}$ given the specific measurement setting $s_{3}$ gives the following lifted CHSH Bell inequality:

$\sum_{x_{1}, x_{2}, a_{1}, a_{2}=0}^{1}(-1)^{a_{1}+a_{2}+x_{1} x_{2}} P\left(a_{1} a_{2} o_{3} \mid x_{1} x_{2} s_{3}\right)-2 P\left(o_{3} \mid s_{3}\right) \stackrel{\mathcal{L}}{\leqslant} 0$.

Lifting the CHSH Bell inequality to an arbitrary number of $n>$ 2 parties can be carried out analogously. In Ref. [39], it was shown that such a procedure not only generates a legitimate Bell inequality but even one that preserves the facet-defining property of the original Bell inequality.

\section{A general recipe for the construction of non- $k$-producible witnesses}

We shall now demonstrate how lifting may be used as a general technique for the construction of Bell-like inequalities for $\mathcal{R}_{n^{\prime}, k}$ starting from one for $\mathcal{R}_{n, k}$ where $n^{\prime}$ is an arbitrary integer greater than $n$ and $\mathcal{R}$ is a resource that respects the nonsignaling constraints. To this end, we note that, without loss of generality, a (linear) Bell-like inequality for a nonsignalingrespecting $\mathcal{R}_{n, k}$ can always be written in the form of

$$
I_{n}=\sum_{\vec{a}, \vec{x}} \beta_{\vec{x}}^{\vec{a}} P(\vec{a} \mid \vec{x}) \stackrel{\mathcal{R}_{n, k}}{\leqslant} 0,
$$

where $\beta_{\vec{x}} \vec{a}$ is some real-valued function of $\vec{a}$ and $\vec{x}$. Our main observation is that the lifting of $I_{n}$ to a scenario involving arbitrary $n^{\prime}>n$ parties is also a legitimate Bell-like inequality for $\mathcal{R}_{n^{\prime}, k}$, as summarized more formally in the following theorem.

Theorem 2. If $I_{n}$ is a Bell-like inequality satisfied by all correlations in $\mathcal{R}_{n, k} \in\{\mathcal{Q}, \mathcal{N S}\}$, i.e., Eq. (16) holds for all $P(\vec{a} \mid \vec{x}) \in \mathcal{R}_{n, k}$, then

$$
I_{n+h}=\sum_{\vec{a}, \vec{x}} \beta_{\vec{x}}^{\vec{a}} P(\vec{a}, \vec{o} \mid \vec{x}, \vec{s}) \stackrel{\mathcal{R}_{n+h, k}}{\leqslant} 0
$$

\footnotetext{
${ }^{8}$ If the distribution vanishes, the conditional distribution given in Eq. (13) is ill-defined.
}

meaning that the lifted inequality holds for all $P(\vec{a}, \vec{o} \mid \vec{x}, \vec{s}) \in$ $\mathcal{R}_{n+h, k}$ where $h \geqslant 1$, while $\vec{o}$ and $\vec{s}$ refer, respectively, to arbitrary but fixed combination of measurement outcomes and measurement settings for the $h$ additional parties.

A proof of this theorem can be found in Appendix C. Clearly, one can see Theorem 2 as a partial generalization of the results presented in [39] from $\mathcal{R}_{n, 1}$ to $\mathcal{R}_{n, k}$ whenever $\mathcal{R} \in\{\mathcal{Q}, \mathcal{N} \mathcal{S}\}$. As for $\mathcal{R} \in\{\mathcal{T}, \mathcal{S}\}$, we know from Corollary 1 and Theorem 2 that any full-correlation Bell-like inequality valid for $\mathcal{R}_{n, k}$ can also be lifted as a Bell-like inequality for $\mathcal{N} \mathcal{S}_{n^{\prime}, k}$ in the extended scenarios. Unfortunately, the theorem in general does not apply to the signaling resource $\mathcal{S}$ (as well as $\mathcal{T}$ ). To see this, consider the tripartite Svetlichny inequality (written in the form given in [20]):

$$
\begin{aligned}
I_{\mathcal{S}, 3} & =\sum_{\vec{x}, \vec{a}} \beta_{\mathcal{S}, 3, \vec{x}}^{\vec{a}} P\left(a_{1} a_{2} a_{3} \mid x_{1} x_{2} x_{3}\right)-4 \stackrel{\mathcal{S}_{3,2}}{\leqslant} 0, \\
\beta_{\mathcal{S}, 3, \vec{x}}^{\vec{a}} & =(-1)^{\sum_{i} a_{i}+\left\lfloor\frac{\sum_{i} x_{i}-1}{2}\right\rfloor} .
\end{aligned}
$$

If Theorem 2 were to be applicable for a Svetlichny resource, we would expect, for instance, that the following inequality,

$$
\begin{aligned}
I_{\mathcal{S}, 4}= & \sum_{\vec{x}, \vec{a}} \beta_{\mathcal{S}, 3, \vec{x}}^{\vec{a}} P\left(a_{1} a_{2} a_{3}, o_{4}=0 \mid x_{1} x_{2} x_{3}, s_{4}=0\right) \\
& -4 \sum_{\vec{a}} P\left(a_{1} a_{2} a_{3}, o_{4}=0 \mid x_{1}^{\prime} x_{2}^{\prime} x_{3}^{\prime}, s_{4}=0\right) \leqslant 0,
\end{aligned}
$$

to hold true for $\mathcal{S}_{4,2}$ and for some arbitrary choice of $x_{1}^{\prime}, x_{2}^{\prime}, x_{3}^{\prime}=$ $\{0,1\}$. One can, however, easily verify that this is not the case. For instance, with $x_{1}^{\prime}=x_{2}^{\prime}=x_{3}^{\prime}=0$, the Svetlichny strategy from $\mathcal{S}_{4,2}$,

$$
\begin{aligned}
& a_{1}=1-\delta_{x_{1}, 1} \delta_{x_{2}, 1}, \quad a_{2}=1, \\
& a_{3}=a_{4}=1-\delta_{x_{3}, 1} \delta_{x_{4}, 0},
\end{aligned}
$$

gives vanishing contribution to the second term in Eq. (19) but an overall value of 4 for $I_{\mathcal{S}, 4}$, clearly violating inequality (19).

Despite the above remark, let us stress once more that there is still wide applicability of Theorem 2. For example, each of the Bell-like inequalities obtained for $\mathcal{N S}_{3,2}$ and $\mathcal{N} \mathcal{S}_{4,2}$ in Refs. [23,31] can now be used to construct witnesses showing MGS $\geqslant 3$ (for the $\mathcal{N S}$ resource) for an arbitrary number of parties. Thanks to Corollary 1 , the families of $k$-partite Svetlichny inequalities obtained in Refs. [19,20] can similarly be extended to detect genuine $\mathcal{N S} k$-partite nonlocality in an arbitrary $n>k$ partite scenario. Likewise, each deviceindependent witness for genuine $k$-partite entanglement obtained in Refs. [20,24,25] can now be applied to witness genuine $k$-partite entanglement in an arbitrary $n>k$ partite scenario. Of course, it remains to show that Bell-like inequalities generated with the help of Theorem 2 could indeed be useful, and this is what we shall show next with a very simple example.

\section{An example where a lifted Bell-like inequality can be used to determine MGS}

Consider the following four-partite mixed state:

$$
\rho=v\left|\mathrm{GHZ}_{3}\right\rangle\left\langle\mathrm{GHZ}_{3}|\otimes| 0\right\rangle\left\langle 0\left|+(1-v) \frac{\mathbb{1}}{2^{3}} \otimes\right| 1\right\rangle\langle 1|,
$$


where $v \in(0,1]$, and $|0\rangle,|1\rangle$ are again the eigenstates of $\sigma_{z}$. Since $\rho$ is biseparable, regardless of which local measurements are performed on $\rho$, the resulting correlations must be in $\mathcal{Q}_{4,3}$ and thus having MGS $\leqslant 3$ for all $\mathcal{R}$ [cf. Eq. (5a)]. Clearly, from Eq. (21), we see that the entanglement of $\rho$ lies entirely within the first three subsystems. Let us denote these systems by $A, B$, and $C$, respectively. For $v \leqslant \frac{1}{5}$, it is known that the tripartite reduced density matrix $\rho_{\mathrm{ABC}}=$ $v\left|\mathrm{GHZ}_{3}\right\rangle\left\langle\mathrm{GHZ}_{3}\right|+(1-v) \frac{\mathbb{1}}{2^{3}}$ is separable [43] and thus not capable of violating any Bell inequalities. Nonetheless, in what follows, we shall show that a lifted Bell-like inequality can indeed be used to show that a certain correlation derived from $\rho$ indeed exhibits MGS $=3$ for all $v \neq 0$, thus showing that the generation of such a correlation quantum mechanically indeed requires at least tripartite entanglement.

To this end, consider now the following dichotomic observables,

$$
\begin{aligned}
& A_{0}=\sigma_{x}, \quad A_{1}=\sigma_{y}, \\
& B_{0}=\frac{1}{\sqrt{2}}\left(\sigma_{x}-\sigma_{y}\right), \quad B_{1}=\frac{1}{\sqrt{2}}\left(\sigma_{x}+\sigma_{y}\right), \\
& C_{0}=-\sigma_{y}, \quad C_{1}=\sigma_{x},
\end{aligned}
$$

and the tripartite Svetlichny inequality given in Eq. (18). It is known that by measuring the local observables $\left\{A_{i}, B_{i}, C_{i}\right\}_{i=0,1}$ given in Eq. (22) on $\left|\mathrm{GHZ}_{3}\right\rangle$, one obtains correlation $\vec{P}$ that violates $I_{\mathcal{S}, 3}$ maximally.

Note that by Corollary 1 and the fact that Eq. (18) is a full-correlation Bell-like inequality, we know that inequality (18) still holds and can be saturated even if we now consider only correlations in $\mathcal{N S}_{3,2}$, i.e.,

$$
I_{\mathcal{N S}, 3}=\sum_{\vec{x}, \vec{a}} \beta_{\mathcal{S}, 3, \vec{x}}^{\vec{a}} P\left(a_{1} a_{2} a_{3} \mid x_{1} x_{2} x_{3}\right)-4 \stackrel{\mathcal{N S} \mathcal{S}_{3,2}}{\leqslant} 0 .
$$

Lifting the inequality $I_{\mathcal{N S}, 3}$ to the specific case where the fourth party performs the zeroth measurement and getting the zeroth outcome, one obtains the inequality:

$$
\begin{aligned}
I_{\mathcal{N S}, 3}^{\mid s_{4}=o_{4}=0}= & \sum_{\vec{x}, \vec{a}} \beta_{\mathcal{N} \mathcal{S}, 3, \vec{x}}^{\vec{a}} P\left(a_{1} a_{2} a_{3}, o_{4}=0 \mid x_{1} x_{2} x_{3}, s_{4}=0\right) \\
& -4 P\left(o_{4}=0 \mid s_{4}=0\right) \stackrel{\mathcal{N} \mathcal{S}_{4,2}}{\leqslant} 0
\end{aligned}
$$

Let us now identify the zeroth measurement of the fourth party by $\sigma_{z}$ and the zeroth outcome by a successful projection onto the eigenstate $|0\rangle$. Together with the measurements specified in Eq. (22), one finds that for all $0<v \leqslant 1$, the resulting correlation derived from $\rho$ must also violate inequality (24). To see this, it suffices to note that (i) for $v \neq 0$, the probability of successfully projecting the fourth system onto $|0\rangle$ is strictly greater than zero and (ii) conditioning on a successful projection, the conditional state for $A B C$ is simply $\left|\mathrm{GHZ}_{3}\right\rangle$ which, as mentioned above, violates $I_{\mathcal{N S}, 3}$ inequality maximally. The aforementioned correlation thus exhibits MGS stronger than that allowed in $\mathcal{N S}_{4.2}$ which, by Eq. (5b), implies that it has MGS $\geqslant 3$ for all $\mathcal{R} \in\{\mathcal{Q}, \mathcal{N S}\}$. Combining this with the biseparability of $\rho$ mentioned above, we see that this particular correlation has exactly $\mathrm{MGS}=3$.

\section{CONCLUSION}

To investigate the extent to which participating parties would need to collaborate nonlocally in a nonlocal game (or equivalently in a Bell-type experiment), we have introduced the notion of minimal group size (MGS), i.e., the smallest number of nonlocally correlated parties required to reproduce a given nonlocal correlation $\vec{P}$. We believe that this more general notion of genuine multipartite nonlocality inspired by $k$ producibility [27] from the studies of multipartite entanglement will be a fruitful approach towards a better understanding of multipartite nonlocality.

As an illustration, we presented, in a four-partite scenario, some genuine tripartite nonlocal correlation where the multipartite nonlocality cannot be detected through the conventional $m$-separability approach. Nonetheless, as first demonstrated in Ref. [16], and further elaborated in this paper, the biseparability approach can in some cases provide tight lower bound on MGS. In fact, for the family of $n$-partite correlations presented in Ref. [16], it was even found that their MGS for a quantum resource is $n$ whereas that for a general nonsignaling (or even an unrestricted signaling) resource is $\left\lceil\frac{n}{2}\right\rceil$, giving an increasing gap between their MGS as $n$ increases. Could there be a bigger gap between the MGS of a nonlocal correlation with respect to a quantum resource and a general (non)signaling resource? In particular, does there exist a multipartite nonlocal quantum correlation which requires genuine $n$-partite entangled state for its production but nonetheless only an MGS of 2 if one is allowed to exploit a signaling, or even a nonsignaling but post-quantum resource? The answer to these questions would certainly shed light on how quantum entanglement helps in a different aspect of communication complexity, namely, how many communicating parties we can replace by quantum entanglement.

We also demonstrated how the technique of lifting [39] originally presented in the context of Bell inequality (for 1-producibility) — can be applied to generate new MGS witnesses starting from one involving a smaller number of parties. This generalizes partially the result of Ref. [39] and provides a useful recipe for the construction of MGS witnesses (with respect to a nonsignaling, e.g., a quantum resource) for an arbitrary $n$-partite scenario. Moreover, we have found that for the complete list of 185 facet-defining Bell-like inequalities of $\mathcal{N S}_{3,2}$ given in Ref. [23], the corresponding MGS witnesses of $\mathcal{N S}_{4,2}$ generated from lifting still correspond to a facet [29] of the polytope in the more complex scenario. Likewise, when these 185 lifted inequalities, as well as the 13479 facet-defining inequalities obtained in Ref. [31] are lifted to the 5-partite scenario, it can again be verified that they correspond to facets of the $\mathcal{N} \mathcal{S}_{5,2}$ polytope. Based on these observations, we conjecture that-as with standard Bell inequalitiesthe procedure of lifting, when applied to a facet-defining inequality of $\mathcal{N} \mathcal{S}_{n, k}$, also generates a facet of $\mathcal{N} \mathcal{S}_{n^{\prime}, k}$ in the extended scenario involving $n^{\prime}>n$ parties.

Unfortunately, a naive application of lifting to signaling resources generally does not always result in legitimate MGS witnesses in the extended scenario. Nevertheless, the possibility to simulate all possible full-correlation functions [20] using only nonsignaling resources - as we show in Appendix Aallows us to apply the recipe to Bell-like inequalities originally 
derived for Svetlichny resources [18-20] and construct MGS witnesses for nonsignaling resources in any extended scenario. It is also conceivable that an analogous witness-generating technique may be found for signaling resources, a problem that we shall leave for future research.

Evidently, on top of Bell-like inequalities that one may construct using the aforementioned technique, it is natural to ask if there exists a simple family of non- $k$-producible witnesses for an arbitrary number of parties. In this regard, we note that a family of such witnesses for a quantum resource (as well as a general nonsignaling resource) has recently been identified [33]. Similar results for other resources, especially one that is either optimal (in the sense of being facet defining) for the respective convex polytope, or one that involves a small number of terms to be measured experimentally, would certainly be desirable.

Finally, let us stress that while we have discussed MGS mostly in the context of reproducing certain nonlocal correlations, these values for the post-quantum nonsignaling resource, as well as for signaling resources also provide insight on the difficulty in reproducing certain correlations using quantum resources. In this sense, evaluation of the MGS for a given correlation may give an indication on how difficult it is to produce certain Bell-inequality violating correlations in the laboratory: The larger the value of MGS, the more systems need to be entangled together in their generation.

\section{ACKNOWLEDGMENTS}

We are very grateful to Jean-Daniel Bancal for sharing with us some arguments related to Sec. III A and for his comments on an earlier version of this manuscript. We also acknowledge many stimulating discussions with Stefano Pironio, especially on his suggestion of a primitive form of the example given in Sec. III D. This work is supported by the Swiss National Centre of Competence in Research Project NCCR-QSIT, the European project CHIST-ERA (DIQIP), and the European Research Council (ERC) Grant No. 258932. F.J.C. acknowledges support from the John Templeton Foundation and the Spanish project FOQUS.

\section{APPENDIX A: PROOF THAT ALL FULL-CORRELATION FUNCTIONS ARE ATTAINABLE USING A NONSIGNALING RESOURCE}

Our goal here is to give a proof that when restricted to the $(\ell-1) m^{n}$-dimensional space of full-correlation functions defined by Eq. (10), the set of legitimate correlations coincides with that achievable by a nonsignaling resource $\mathcal{N S}_{n}$. To this end, it is worth remembering that the set of normalized correlations in this space is precisely the set of correlations achievable by the Svetlichny resource $\mathcal{S}_{n}$. To prove the desired result, it is then sufficient to show that all extreme points of $\mathcal{S}_{n}$ in this space are also achievable using $\mathcal{N S}_{n}$.

Proof. First, let us note that all extremal strategies of these full-correlation functions are deterministic functions of the joint inputs $\vec{x}$, i.e., they are defined by specifying for each given $\vec{x}$, the corresponding sum of outputs modulo $\ell$. In other words, for each of these extremal strategies and for each given $\vec{x}$, we have

$$
P\left(\left[\mathbf{a}_{\vec{x}}\right]_{k}=r\right)=\delta_{r, f(\vec{x})},
$$

where $f(\vec{x})$ is some deterministic, $r$-value function of $\vec{x}$. Different extremal strategies of $\mathcal{S}_{n}$ in this space then correspond to different choices of $f(\vec{x})$. To prove Theorem 1 , it is then sufficient to find a nonsignaling strategy that gives Eq. (A1) for an arbitrary choice of $f(\vec{x})$.

Let us first illustrate how this works in the scenario of $n=2$. Consider the following normalized probability distribution:

$$
P\left(a_{1} a_{2} \mid x_{1} x_{2}\right)=\frac{1}{\ell} \delta_{a_{1}+a_{2} \bmod \ell, f\left(x_{1}, x_{2}\right)} .
$$

Note that (regardless of $x_{1}$ and $x_{2}$ ) for each $a_{1}$-due to the Kronecker delta-there is one, and only one value of $a_{2}$ such that the right-hand side of Eq. (A2) is nonvanishing, likewise for $a_{2}$. As a result, the corresponding marginal distributions are given by

$$
\begin{aligned}
& P\left(a_{1} \mid x_{1} x_{2}\right)=\sum_{a_{2}} \frac{1}{\ell} \delta_{a_{1}+a_{2} \bmod \ell, f\left(x_{1}, x_{2}\right)}=\frac{1}{\ell}, \\
& P\left(a_{2} \mid x_{1} x_{2}\right)=\sum_{a_{1}} \frac{1}{\ell} \delta_{a_{1}+a_{2} \bmod \ell, f\left(x_{1}, x_{2}\right)}=\frac{1}{\ell} .
\end{aligned}
$$

Both these marginal distributions are independent of the input of the other party and hence the distribution given in Eq. (A2) satisfies the nonsignaling constraints. From these observations and Eq. (10b), it is also easy to see that the nonsignaling distribution given in Eq. (A2) satisfies Eq. (A1). We have thus shown that in the above-mentioned subspace of full-correlation functions, the extremal strategy of $\mathcal{S}_{2}$ can also be achieved by a nonsignaling correlation. More generally, for arbitrary $n \geqslant 2$, it is easy to verify that the following distribution,

$$
P(\vec{a} \mid \vec{x})=\frac{1}{\ell^{n-1}} \delta_{\sum_{i} a_{i} \bmod \ell, f(\vec{x})}
$$

is nonsignaling, giving a uniform $n^{\prime \prime}$-partite marginal distribution of $\ell^{-n^{\prime \prime}}$, and satisfies Eq. (A1). In other words, we have proved that the extremal strategy of $\mathcal{S}_{n}$ in the subspace of full-correlation functions can always be achieved using a nonsignaling strategy.

\section{APPENDIX B: PROOF OF COROLLARY 1}

Here, we give a proof of Corollary 1. For concreteness, we shall provide a proof for $\mathcal{R}=\mathcal{S}$. The case for $\mathcal{R}=\mathcal{T}$ follows from the inclusion relations given in Eq. (4).

Proof. Given inequality (11), the inclusion relations of Eq. (4) immediately imply that inequality (12) holds true for all $\mathcal{P}(\vec{a} \mid \vec{x}) \in \mathcal{N} \mathcal{S}_{n, k}$. It thus remains to show that there also exists $P(\vec{a} \mid \vec{x})=P_{0}^{\mathcal{N} S}(\vec{a} \mid \vec{x}) \in \mathcal{N} \mathcal{S}_{n, k}$ such that the inequality (12) is saturated, i.e.,

$$
\sum_{\vec{x}} \sum_{r=0}^{\ell-1} \beta_{\vec{x}}^{r} P_{0}^{\mathcal{N} \mathcal{S}}\left(\left[\mathbf{a}_{\vec{x}}\right]_{\ell}=r\right)=B_{n, k}^{\mathcal{S}} .
$$


By assumption, there exists extremal $P(\vec{a} \mid \vec{x})=P^{\mathcal{S}}(\vec{a} \mid \vec{x}) \in$ $\mathcal{S}_{n, k}$ such that inequality (11) is saturated, i.e.,

$$
\sum_{\vec{x}} \sum_{r=0}^{\ell-1} \beta_{\vec{x}}^{r} P^{\mathcal{S}}\left(\left[\mathbf{a}_{\vec{x}}\right]_{\ell}=r\right)=B_{n, k}^{\mathcal{S}} .
$$

From the definition of the full-correlation function, Eq. (10b), and the assumed $k$ producibility of the correlation, we have

$$
\begin{aligned}
P^{\mathcal{S}}\left(\left[\mathbf{a}_{\bar{x}}\right]_{\ell}=r\right) & =\sum_{\vec{a}} P^{\mathcal{S}}(\vec{a} \mid \vec{x}) \delta_{\left[\mathbf{a}_{\bar{x}}\right]_{\ell}, r} \\
& =\sum_{\vec{a}} \prod_{i=1}^{G} P^{\mathcal{S}}\left(\vec{a}^{[i]} \mid \vec{x}^{[i]}\right) \delta_{\left[\mathbf{a}_{\vec{x}}\right]_{\ell}, r},
\end{aligned}
$$

where $P^{\mathcal{S}}\left(\vec{a}^{[i]} \mid \vec{x}^{[i]}\right)$ refers to the $i$ th constituent distribution, which is at most $k$ partite. Denote the sum of the outputs in the $j$ th group by $\mathbf{a}_{\vec{x}[j]}$; we can then further rewrite $P^{\mathcal{S}}\left(\left[\mathbf{a}_{\vec{x}}\right]_{\ell}=r\right)$ as

$$
\begin{aligned}
& \prod_{i=1}^{G} \sum_{\vec{a}^{[i]}} P^{\mathcal{S}}\left(\vec{a}^{[i]} \mid \vec{x}^{[i]}\right) \delta_{\left[\sum_{j}\left[\mathbf{a}_{\bar{x}}[j]\right]_{e}\right] \in, r}, \\
& \quad=\prod_{i=1}^{G} \sum_{\vec{a}^{[i]}} \sum_{r^{[i]}=0}^{\ell-1} P^{\mathcal{S}}\left(\vec{a}^{[i]} \mid \vec{x}^{[i]}\right) \delta_{\left[\mathbf{a}_{\vec{x}}[i]\right]_{e}, r^{[i]}} \delta_{\left[\sum_{j}\left[\mathbf{a}_{\bar{x}}[j]\right]_{e}\right]_{e}, r .} .
\end{aligned}
$$

Note that for each $\vec{x}^{[i]}$, due to the Kronecker delta $\delta_{\left[\mathbf{a}_{[i[}\right]_{e}, r^{[i]}}$, there is only one term in the sum over $r^{[i]}$ that contributes nontrivially. Swapping the order of the sums gives

$$
\begin{aligned}
& \prod_{i=1}^{G} \sum_{r^{[i]}=0}^{\ell-1} \sum_{\vec{a}^{[i]}} P^{\mathcal{S}}\left(\vec{a}^{[i]} \mid \vec{x}^{[i]}\right) \delta_{\left[\mathbf{a}_{\bar{x}}[i]\right] \ell}, r^{[i]} \delta_{\left[\sum_{j}\left[\mathbf{a}_{\bar{x}}[j]\right]_{\ell}\right]_{\ell}, r}, \\
& =\prod_{i=1}^{G} \sum_{r^{[i]}=0}^{\ell-1} \sum_{\vec{a}^{[i]}} P^{\mathcal{S}}\left(\vec{a}^{[i]} \mid \vec{x}^{[i]}\right) \delta_{\left[a_{\vec{x}}[i]\right]_{\ell}, r^{[i]}} \delta_{\left[\sum_{j} r^{[j]}\right]_{\ell}, r}, \\
& =\prod_{i=1}^{G} \sum_{r^{[i]}=0}^{\ell-1} P^{\mathcal{S}}\left(\left[\mathbf{a}_{\vec{x}^{[i]}}\right]_{\ell}=r^{[i]}\right) \delta_{\left[\sum_{j} r^{[j]}\right]_{\ell}, r},
\end{aligned}
$$

which means that $P^{\mathcal{S}}\left(\left[\mathbf{a}_{\vec{x}}\right]_{\ell}=r\right)$ factorizes into a (linear combination of) product of full-correlation functions for each group $P^{\mathcal{S}}\left(\left[\mathbf{a}_{\vec{x}^{[i]}}\right]_{\ell}=r^{[i]}\right)$. By Theorem 1 , there is no loss of generality in replacing the constituent distribution from the $i$ th group $P^{\mathcal{S}}\left(\vec{a}^{[i]} \mid \vec{x}^{[i]}\right)$ by some nonsignaling distributions $P_{0}^{\mathcal{N} \mathcal{S}}\left(\vec{a}^{[i]} \mid \vec{x}^{[i]}\right)$ such that they agree at the level of the fullcorrelation functions, i.e.,

$$
P^{\mathcal{S}}\left(\left[\mathbf{a}_{\vec{x}^{[i]}}\right]_{\ell}=r^{[i]}\right)=P_{0}^{\mathcal{N S}}\left(\left[\mathbf{a}_{\vec{x}^{[i]}}\right]_{\ell}=r^{[i]}\right) \quad \forall i, r^{[i]} .
$$

Substituting this back into Eq. (B3) and then Eq. (B2), we thus obtain Eq. (B1) by identifying

$$
P_{0}^{\mathcal{N S}}\left(\left[\mathbf{a}_{\vec{x}^{[i]}}\right]_{\ell}=r^{[i]}\right)=\sum_{\vec{a}} \prod_{i=1}^{G} P^{\mathcal{N S}}\left(\vec{a}^{[i]} \mid \vec{x}^{[i]}\right) \delta_{\left[\mathbf{a}_{\vec{x}}\right]_{\ell}, r} .
$$

An immediate consequence of the above corollary is that any full-correlation Bell-like inequality for $\mathcal{S}_{n, k}$, such as those derived in Refs. [18-20], is also valid and tight for $\mathcal{N S}_{n, k}$.

\section{APPENDIX C: PROOF OF THEOREM 2}

We now provide a proof of Theorem 2.

Proof. By assumption, the following expression holds true:

$$
I_{n}=\sum_{\vec{a}, \vec{x}} \beta_{\vec{x}}^{\vec{a}} P(\vec{a} \mid \vec{x}) \leqslant 0,
$$

for all $P(\vec{a} \mid \vec{x}) \in \mathcal{R}_{n, k}$, and our goal is to show that

$$
I_{n+h}=\sum_{\vec{a}, \vec{x}} \beta_{\vec{x}}^{\vec{a}} P(\vec{a}, \vec{o} \mid \vec{x}, \vec{s}) \stackrel{\mathcal{R}_{n+h, k}}{\leqslant} 0,
$$

for arbitrary $h \geqslant 1$ and all fixed choices of $\vec{o}$ and $\vec{s}$. We will show that this is the case by reductio ad impossibilem.

Suppose the converse, namely, that there exists some choice of $\vec{o}, \vec{s}$, and $h$ such that for some $P(\vec{a}, \vec{o} \mid \vec{x}, \vec{s}) \in \mathcal{R}_{n+h, k}$,

$$
\sum_{\vec{a}, \vec{x}} \beta_{\vec{x}}^{\vec{a}} P(\vec{a}, \vec{o} \mid \vec{x}, \vec{s})>0 .
$$

By linearity of the expression and the requirement that $P(\vec{a}, \vec{o} \mid \vec{x}, \vec{s}) \in \mathcal{R}_{n+h, k}$, the above inequality implies that there exists some correlation,

$$
P(\vec{a}, \vec{o} \mid \vec{x}, \vec{s})=\prod_{i=1}^{G} P^{\mathcal{R}}\left(\vec{a}^{[i]}, \vec{o}^{[i]} \mid \vec{x}^{[i]}, \vec{s}^{[i]}\right),
$$

such that

$$
\sum_{\vec{a}, \vec{x}} \beta_{\vec{x}}^{\vec{a}} \prod_{i=1}^{G} P^{\mathcal{R}}\left(\vec{a}^{[i]}, \vec{o}^{[i]} \mid \vec{x}^{[i]}, \vec{s}^{[i]}\right)>0,
$$

where $P^{\mathcal{R}}\left(\vec{a}^{[i]}, \vec{o}^{[i]} \mid \vec{x}^{[i]}, \vec{s}^{[i]}\right)$ refers to the $i$ th constituent distribution (from the $i$ th group), and as with $P(\vec{a}, \vec{o} \mid \vec{x}, \vec{s})$, we have used $\vec{o}^{[i]}$ and $\vec{s}^{[i]}$ to indicate, respectively, the (possibly empty) outcome and setting string that are fixed in $P^{\mathcal{R}}\left(\vec{a}^{[i]}, \vec{o}^{[i]} \mid \vec{x}^{[i]}, \vec{s}^{[i]}\right)$. Note that the assumption of $P(\vec{a}, \vec{o} \mid \vec{x}, \vec{s}) \in \mathcal{R}_{n+h, k}$ implies that each constituent distribution is at most $k$ partite and their respective size $n_{i}$ sums up to $n+h$, i.e., $\sum_{i=1}^{G} n_{i}=n+h$.

Evidently, since inequality (C5) is strict and that $P^{\mathcal{R}}\left(\vec{a}^{[i]}, \vec{o}^{[i]} \mid \vec{x}^{[i]}, \vec{s}^{[i]}\right) \geqslant 0$ for all $\vec{a}^{[i]}$ and $\vec{x}^{[i]}$, it must be the case that

$$
\sum_{\vec{a}^{[i]}} P^{\mathcal{R}}\left(\vec{a}^{[i]}, \vec{o}^{[i]} \mid \vec{x}^{[i]}, \vec{s}^{[i]}\right)>0,
$$

for all $\vec{x}^{[i]}$ that contribute nontrivially in the left-hand side of Eq. (C5). In fact, since the left-hand side of inequality (C6) can also be obtained by performing the appropriate sums of Eq. (C4),

$$
\begin{aligned}
\sum_{\vec{a}, \vec{o}[j]} P(\vec{a}, \vec{o} \mid \vec{x}, \vec{s}) & =\sum_{\vec{a}, \vec{o}^{[j]} \mid j \neq i} \prod_{\ell=1}^{G} P^{\mathcal{R}}\left(\vec{a}^{[\ell]}, \vec{o}^{[\ell]} \mid \vec{x}^{[\ell]}, \vec{s}^{[\ell]}\right) \\
& =\sum_{\vec{a}^{[i]}} P^{\mathcal{R}}\left(\vec{a}^{[i]}, \vec{o}^{[i]} \mid \vec{x}^{[i]}, \vec{s}^{[i]}\right),
\end{aligned}
$$

we see that by the nonsignaling nature of $P(\vec{a}, \vec{o} \mid \vec{x}, \vec{s})$, the very last expression of Eq. (C7) must also be independent of $\vec{x}^{[i]}$. Hereafter, we shall simply write these marginal distributions 
as

$$
P^{\mathcal{R}}\left(\vec{o}^{[i]} \mid \vec{s}^{[i]}\right)=\sum_{\vec{a}^{[i]}} P^{\mathcal{R}}\left(\vec{a}^{[i]}, \vec{o}^{[i]} \mid \vec{x}^{[i]}, \vec{s}^{[i]}\right) .
$$

Hence, from inequality (C6), we see that the conditional distributions,

$$
\tilde{P}^{\mid \vec{o}^{[i,}, \vec{s}^{[i]}}\left(\vec{a}^{[i]} \mid \vec{x}^{[i]}\right)=\frac{P^{\mathcal{R}}\left(\vec{a}^{[i]}, \vec{o}^{[i]} \mid \vec{x}^{[i]}, \vec{s}^{[i]}\right)}{P^{\mathcal{R}}\left(\vec{o}^{[i]} \mid \vec{s}^{[i]}\right)},
$$

are well defined for all $\vec{x}^{[i]}$ and satisfy the normalization

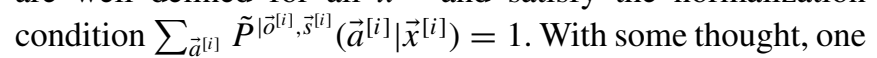
can also see that the conditional distribution defined in Eq. (C9) also inherits the property of the defining distribution, i.e., satisfying the constraint defined by $\mathcal{R}$. For instance, if
$P^{\mathcal{R}}\left(\vec{a}^{[i]}, \vec{o}^{[i]} \mid \vec{x}^{[i]}, \vec{s}^{[i]}\right)$ admits a quantum representation, so does $\tilde{P} \tilde{P}^{\left[\hat{o}^{[i]}, \hat{s}^{[i]}\right.}\left(\vec{a}^{[i]} \mid \vec{x}^{[i]}\right)$.

Dividing inequality (C5) by $\prod_{i} P^{\mathcal{R}}\left(\vec{o}^{[i]} \mid \vec{s}^{[i]}\right)$ and using Eq. (C9), we obtain

$$
\sum_{\vec{a}, \vec{x}} \beta_{\vec{x}}^{\vec{a}} \prod_{i=1}^{G} \tilde{P}^{\left[\left.\right|^{[i]}, \vec{s}^{[i]}\right.}\left(\vec{a}^{[i]} \mid \vec{x}^{[i]}\right)>0 .
$$

As mentioned above, for all $i$, the conditional distribution

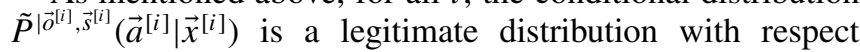
to the resource $\mathcal{R}$ and cannot be more than $k$ partite, i.e, $\prod_{i=1}^{G} \tilde{P}^{\left[\left.\right|^{[i]}, \hat{s}^{[i]}\right.}\left(\vec{a}^{[i]} \mid \vec{x}^{[i]}\right) \in \mathcal{R}_{n, k}$. Hence, inequality (C10) implies that the original inequality $I_{n}$ can be violated by correlation in $\mathcal{R}_{n, k}$, which contradicts our very first assumption that $I_{n}$ is a legitimate Bell-like inequality for $\mathcal{R}_{n, k}$.
[1] J. S. Bell, Physics (NY) 1, 195 (1964).

[2] R. Cleve, P. Høyer, B. Toner, and J. Watrous, in Proceedings of the 19th IEEE Annual Conference on Computational Complexity 2004, Amherst, MA (IEEE Conference Proceedings, New York, 2004), pp. 236-249.

[3] A. C. Doherty, Y.-C. Liang, B. Toner, and S. Wehner, in Proceedings of the 23rd IEEE International Conference on Computational Complexity (IEEE Computer Society, College Park, 2008), pp. 199-210.

[4] H. Buhrman, R. Cleve, and W. van Dam, SIAM J. Comput. 30, 1829 (2001); Č. Brukner, M. Żukowski, J.-W. Pan, and A. Zeilinger, Phys. Rev. Lett. 92, 127901 (2004).

[5] J. Barrett, L. Hardy, and A. Kent, Phys. Rev. Lett. 95, 010503 (2005); A. Acín, N. Gisin, and Ll. Masanes, ibid. 97, 120405 (2006); A. Acín, N. Brunner, N. Gisin, S. Massar, S. Pironio, and V. Scarani, ibid. 98, 230501 (2007).

[6] R. Colbeck, Ph.D thesis, University of Cambridge, 2006; S. Pironio et al., Nature (London) 464, 1021 (2010).

[7] N. Brunner, D. Cavalcanti, S. Pironio, V. Scarani, and S. Wehner, Rev. Mod. Phys. 86, 419 (2014).

[8] B. Coecke. T. Fritz, and R. W. Spekkens, arXiv:1409.5531.

[9] B. F. Toner and D. Bacon, Phys. Rev. Lett. 91, 187904 (2003); O. Regev and B. Toner, SIAM J. Comput. 39, 1562 (2009).

[10] N. J. Cerf, N. Gisin, S. Massar, and S. Popescu, Phys. Rev. Lett. 94, 220403 (2005).

[11] J. Barrett, N. Linden, S. Massar, S. Pironio, S. Popescu, and D. Roberts, Phys. Rev. A 71, 022101 (2005).

[12] N. S. Jones and Ll. Masanes, Phys. Rev. A 72, 052312 (2005).

[13] J. Barrett and S. Pironio, Phys. Rev. Lett. 95, 140401 (2005).

[14] A. Broadbent, P. R. Chouha and A. Tapp, in Proceedings of the 3rd International Conference on Quantum, Nano and Micro Technologies Mexico (IEEE Computer Society, Cancun, Mexico, 2009), pp. 59-62; J.-D. Bancal, C. Branciard, and N. Gisin, Adv. Math. Phys. 2010, 293245 (2010); C. Branciard and N. Gisin, Phys. Rev. Lett. 107, 020401 (2011); G. Brassard and M. Kaplan, Lect. Notes Comput. Sci. 7582, 65 (2013); G. Brassard, L. Devroye, and C. Gravel, arXiv:1303.5942.

[15] B. Toner, Proc. R. Soc. A 465, 59 (2009); Ll. Masanes, A. Acín, and N. Gisin, Phys. Rev. A 73, 012112 (2006); B. Toner and F. Verstraete, arXiv:cond-mat/0611001; M. P. Seevinck, Quant. Inf. Proc. 9, 273 (2010); P. Kurzyński, A. Cabello, and D. Kaszlikowski, Phys. Rev. Lett. 112, 100401 (2014); R. Ramanathan and P. Horodecki, ibid. 113, 210403 (2014).

[16] Y.-C. Liang, F. J. Curchod, J. Bowles, and N. Gisin, Phys. Rev. Lett. 113, 130401 (2014).

[17] J.-D. Bancal, C. Branciard, N. Gisin, and S. Pironio, Phys. Rev. Lett. 103, 090503 (2009).

[18] G. Svetlichny, Phys. Rev. D 35, 3066 (1987).

[19] D. Collins, N. Gisin, S. Popescu, D. Roberts, and V. Scarani, Phys. Rev. Lett. 88, 170405 (2002); M. Seevinck and G. Svetlichny, ibid. 89, 060401 (2002); N. S. Jones, N. Linden, and S. Massar, Phys. Rev. A 71, 042329 (2005); J.-D. Bancal, N. Brunner, N. Gisin, and Y.-C. Liang, Phys. Rev. Lett. 106, 020405 (2011); J.-L. Chen, D.-L. Deng, H.-Y. Su, C. Wu, and C. H. Oh, Phys. Rev. A 83, 022316 (2011).

[20] J.-D. Bancal, C. Branciard, N. Brunner, N. Gisin, and Y.-C. Liang, J. Phys. A: Math. Theor. 45, 125301 (2012).

[21] L. Aolita, R. Gallego, A. Cabello, and A. Acín, Phys. Rev. Lett. 108, 100401 (2012).

[22] R. Gallego, L. E. Würflinger, A. Acín, and M. Navascués, Phys. Rev. Lett. 109, 070401 (2012).

[23] J.-D. Bancal, J. Barrett, N. Gisin, and S. Pironio, Phys. Rev. A 88, 014102 (2013).

[24] J.-D. Bancal, N. Gisin, Y.-C. Liang, and S. Pironio, Phys. Rev. Lett. 106, 250404 (2011).

[25] K. F. Pál and T. Vértesi, Phys. Rev. A 83, 062123 (2011).

[26] T. Moroder, J.-D. Bancal, Y.-C. Liang, M. Hofmann, and O. Gühne, Phys. Rev. Lett. 111, 030501 (2013).

[27] O. Gühne, G. Tóth, and H. J. Briegel, New J. Phys. 7, 229 (2005).

[28] S. Popescu and D. Rohrlich, Found. Phys. 24, 379 (1994).

[29] B. Grúnbaum, Convex Polytopes (Springer, New York, 2004).

[30] S. Boyd and L. Vandenberghe, Convex Optimization (Cambridge, New York, 2004).

[31] F. J. Curchod, Y.-C. Liang, and N. Gisin, J. Phys. A: Math. Theo. 47, 424014 (2014).

[32] F. J. Curchod, Master thesis, University of Geneva, 2013.

[33] Y.-C. Liang, D. Rosset, J.-D. Bancal, G. Pütz, T. J. Barnea, and N. Gisin, arXiv:1411.7385.

[34] D. M. Greenberger, M. A. Horne, A. Zeilinger, in Bell's Theorem, Quantum Theory, and Conceptions of the Universe, edited by M. Kafatos (Kluwer, Dordrecht, 1989), pp. 69-72; N. D. Mermin, Phys. Rev. Lett. 65, 3373 (1990).

[35] C. Śliwa, Phys. Lett. A 317, 165 (2003). 
[36] N. D. Mermin, Phys. Rev. Lett. 65, 1838 (1990); M. Ardehali, Phys. Rev. A 46, 5375 (1992).

[37] S. M. Roy and V. Singh, Phys. Rev. Lett. 67, 2761 (1991); A. V. Belinskiı̌ and D. N. Klyshko, Phys. Usp. 36, 653 (1993); N. Gisin and H. Bechmann-Pasquinucci, Phys. Lett. A 246, 1 (1998).

[38] K. Nagata, M. Koashi, and N. Imoto, Phys. Rev. Lett. 89, 260401 (2002).

[39] S. Pironio, J. Math. Phys. 46, 062112 (2005).
[40] M. T. Quintino, Master thesis, Universidade Federal de Minas Gerais, 2012.

[41] J.-D. Bancal, S. Pironio, A. Acín, Y.-C. Liang, V. Scarani, and N. Gisin, Nature Phys. 8, 867 (2012); T. J. Barnea, J.-D. Bancal, Y.-C. Liang, and N. Gisin, Phys. Rev. A 88, 022123 (2013).

[42] J. F. Clauser, M. A. Horne, A. Shimony, and R. A. Holt, Phys. Rev. Lett. 23, 880 (1969).

[43] W. Dür and J. I. Cirac, Phys. Rev. A 61, 042314 (2000). 